\title{
Thermal and optical properties of electron beam irradiated cellulose triacetate
}

\author{
S A Nouh ${ }^{1 *}$, Amal Mohamed ${ }^{2}$ and $\mathrm{H} \mathrm{M} \mathrm{El} \mathrm{Hussieny}{ }^{1}$ \\ ${ }^{1}$ Department of Physics, Faculty of Science, Ain Shams University, Cairo, Egypt \\ ${ }^{2}$ Department of Physics, Faculty of Science, Zagazig University, Cairo, Egypt \\ E-mail : samirnouh@yahoo.com
}

\begin{abstract}
Samples from Cellulose triacetate (CTA) sheets were irradiated with electron beam in the dose range 10-200 kGy. Non-isothermal studies were carried out using thermogravimetric analysis (TGA) to obtain the activation energy of thermal decomposition for CTA polymer. The CTA samples decompose in one main break down stage. The results indicate that the irradiation by electron beam in the dose range 80-200 kGy increases the thermal stability of the polymer samples. Also, the variation of melting temperatures with the electron dose has been determined using differential thermal analysis (DTA). The CTA polymer is characterized by the appearance of one endothermic peak due to melting. It is found that the irradiation in the dose range 10-80 kGy causes defects generation that splits the crystals depressing the melting temperature, while at higher doses (80-200 kGy), the thickness of crystalline structure (lamellae) is increased, thus the melting temperature increases. In addition, the transmission of these samples in the wavelength range $200-2500 \mathrm{~nm}$, as well as any color changes, were studied. The color intensity $\Delta E^{*}$ was greatly increased on increasing the electron beam dose, and accompanied by a significant increase in the blue color component.
\end{abstract}

Keywords : Electron beam irradiation, thermal properties, color response, polymers.

PACS No. : 78.20.-e

\section{Introduction}

Radiation modification of polymers is one of the modern methods, which leads to new polymeric materials with specific properties. Also, electron-beam irradiation can be considered as one of the most popular and well established processes for several applications [1]. The action of electron beam rays on polymers leads to several changes in the polymer properties due to the induced chain scissions and cross-links. The degradation induced by electron beam irradiation is a prompt way to simulate the aging of polymeric materials and to study their radiation stability or the changes in physical properties in view of their industrial applications [2,3]. Also, polymers can be easily affected by the variations of temperature. In fact, such effects would induce modifications in the chain segment mobility of polymers, indicated by changes in 
transition temperatures [4,5]. Cellulose triacetate is one of the polymers that his been usefully employed in a number of different fields of science and technology [6]. Several investigations have been introduced to study the changes in physical properties of polymers due to irradiation [7-14]. The aim of the present study is to obtain information concerning the interaction of electron beam rays with cellulose triacetate polymer to improve its performance in several industrial applications.

\section{Experimental}

\subsection{Samples :}

Cellulose triacetate polymer used in this study is $0.25 \mathrm{~mm}$ thick sheet manufactured by Eastman Kodak Company, Rochester, New York.

\subsection{Irradiation facilities :}

The irradiation process was performed in air, at room temperature $25^{\circ} \mathrm{C}$, using $1.5 \mathrm{MeV}$ electron beam accelerator of the ICT-type. It operates with insulating core transformer, with a beam current of $25 \mathrm{~mA}$. The conveyer was attached with a cooling system to avoid heating of the samples. The method of irradiating polymer films comprises in multiple irradiations at $10 \mathrm{kGy}$ per pass. The dose was adjusted frequently using FWT'60-00 dosimeter that was calibrated by irradiation in gamma facility against Ceric/Cerous dosimeter supplied by Nordion, Canada. It is recognized that transfer of the calibration from gamma to $1.5 \mathrm{MeV}$ electron beam irradiation involves an added uncertainty. We estimate this uncertainty to be less than $5 \%$.

\subsection{Experimental apparatus :}

The thermal behavior was investigated using differential thermal analysis (DTA) and thermogravimetric analysis (TGA) with a type Shimadzu-50 instrument. $\alpha-\mathrm{Al}^{2} \mathrm{O}^{3}$ powder was used as a reference for DTA measurements. Thermal experiments were carried out at a heating rate of $10^{\circ} \mathrm{C} / \mathrm{min}$ with $\mathrm{N}_{2}$ as a carrier gas at a flow rate of $30 \mathrm{~cm}^{3} / \mathrm{min}$.

The transmission measurements were carried out using a Shimadzu UV-Vis-Nir scanning spectrophotometer, type $3101 \mathrm{PC}$. This unit measures in the wavelength range 200-3000 nm. The CIE (Commission International De E' Claire units $x, y$, and $z$ ) approach was used in the present work for the description of colored samples. The $L^{*}$, $a^{*}, b^{*}$ intercepts used in this system are based on the CIE color triangle. In this system, the $L^{*}$ value specifies the dark-white axis, $a^{*}$ the green-red axis, and $b^{*}$ the blue-yellow axis. The $L^{*}, a^{*}, b^{*}$ intercepts of CTA films were measured and taken as a reference. The color difference $\left(\Delta E^{*}\right)$ between the non irradiated CTA sample and those irradiated with different doses was calculated according to the CIELAB colordifference equation $[15,16]$ :

$$
\Delta E^{*}=\left[\left(\Delta L^{*}\right)^{2}+\left(\Delta a^{*}\right)^{2}+\left(\Delta b^{*}\right)^{2}\right]^{1 / 2}
$$




\section{Results and discussion}

\subsection{Thermal properties :}

\subsubsection{Thermogravimetric analysis (TGA):}

Thermogravimetric analysis (TGA) is a technique that measures the change in weight of a sample during heating. It provides information on the initiation and termination of weight change and the amount of change. TGA was performed for irradiated and non irradiated CTA samples in the temperature range from room temperature up to $600^{\circ} \mathrm{C}$, at a heating rate of $10^{\circ} \mathrm{C} / \mathrm{min}$. It is found that the CTA polymer decomposes in one main weight loss stage. Using these TGA thermograms, the values of onset temperature of decomposition $T_{0}$ (the temperature at which the thermal decomposition starts) were calculated and are given in Table 1.

Table 1. Values of the onset temperature of decomposition $T_{0}$, activation energy of thermal decomposition $E_{a}$ and melting temperature $T_{m}$ for CTA samples as a function of the electron dose.

\begin{tabular}{cccc}
\hline $\begin{array}{c}\text { Electron dose } \\
\text { (kGy) }\end{array}$ & $T_{0}\left({ }^{\circ} \mathrm{C}\right)$ & $E_{a}(\mathrm{eV})$ & $T_{m}\left({ }^{\circ} \mathrm{C}\right)$ \\
\hline 0 & 177 & 1.86 & 323 \\
10 & 166 & 1.62 & 319 \\
20 & 162 & 1.31 & 322 \\
40 & 156 & 1.05 & 314 \\
80 & 149 & 0.88 & 306 \\
100 & 159 & 1.09 & 310 \\
130 & 179 & 1.18 & 314 \\
160 & 189 & 1.61 & 317 \\
200 & 192 & 1.74 & 319 \\
\hline
\end{tabular}

Figure 1 shows the variation of $T_{0}$ with the electron dose. The figure shows that $T_{0}$ decreases until a minimum value around the $80 \mathrm{kGy}$ irradiated sample indicating a

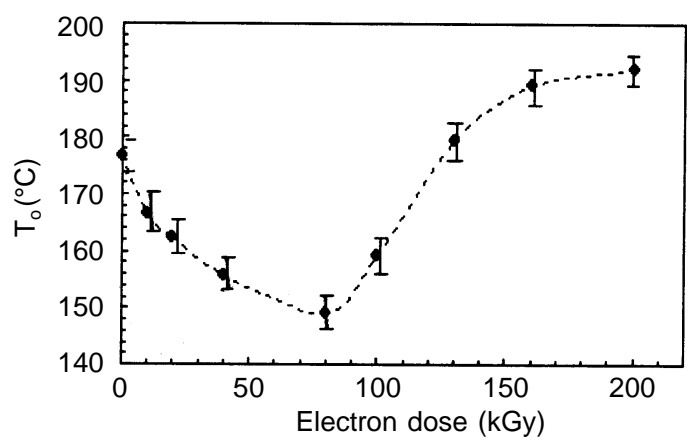

Figure 1. Variation of onset temperature of decomposition $T_{0}$ with the electron dose. 
decrease in thermal stability of the polymer samples due to degradation (i.e. preferentially chain scission), then increases on increasing the electron dose up to 200 kGy due to cross-linking process.

\subsubsection{Activation energy of thermal decomposition :}

Not only TGA gives the ability to find out the temperature at which the thermal decomposition starts $T_{0}$ but also allows the measurement of the activation energy of thermal decomposition $E_{a}$, which is useful for studying the thermal stability of the materials. The method proposed by Horowitz and Metzger [17] has been used in the present study for the measurements of the thermal activation energies. In this method TG curves obtained at a heating rate of $10^{\circ} \mathrm{C} / \mathrm{min}$ are required where the following equation is valid :

$$
\ln \left\{\ln \left[\left(W_{0}-W_{f}\right) /\left(W-W_{f}\right)\right]\right\}=E_{a} \theta / R T_{s}^{2}
$$

where $R$ is the general gas constant, $W_{0}$ and $W_{f}$ are the initial and final weights of the stage, $W$ is the remaining weight at a given temperature $T, \theta$ is the temperature difference between $T$ and $T_{s}$.

According to the above equation, a plot of $\ln \left\{\ln \left[\left(W_{0}-W_{f}\right) /\left(W-W_{f}\right)\right]\right\}$ against $\theta$ leads to a straight-line relationship in the range where the decomposed ratios are equal. Hence, the activation energy of thermal decomposition $E_{a}$ can be evaluated from the slope of the line. $T_{s}$ is the temperature which satisfies the equation :

$$
\left[\left(W-W_{f}\right) /\left(W_{0}-W_{f}\right)\right]=(1 / e)=0.3679
$$

Using the TGA curves, values of activation energy of thermal decomposition $E_{a}$ were calculated and are given in Table 1. Figure 2 shows the variation of $E_{a}$ with the electron dose. From the figure it is clear that $E_{a}$ decreases until a minimum value around the $80 \mathrm{kGy}$ irradiated sample due to chain scission, followed by an increase on increasing the electron dose up to $200 \mathrm{kGy}$ due to crosslinking mechanism.

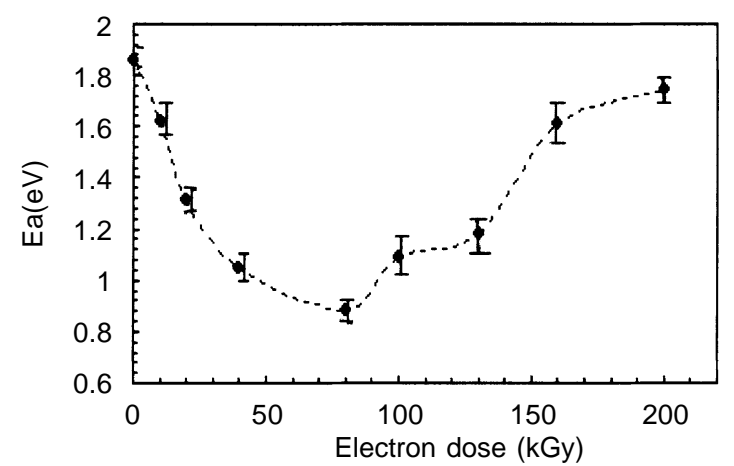

Figure 2. Variation of activation energy of thermal decomposition $E_{a}$ with the electron dose.

\subsubsection{Differential thermal analysis (DTA) :}

Differential thermal analysis DTA was performed, in the temperature range from room 
temperature up to $400^{\circ} \mathrm{C}$, at a heating rate of $10^{\circ} \mathrm{C} / \mathrm{min}$ on the CTA samples. All the thermograms were characterized by the appearance of one endothermic peak at the melting temperature. On heating, the samples pass through a range of badly specified softening temperatures. This can be attributed to the fact that any polymeric chain has more degree of freedom than non-polymeric matter, and there is variability in chain length. Approximate indicative values of these melting temperatures were calculated and are given in Table 1. The values obtained indicate that the melting temperature $T_{m}$ almost decreases until a minimum value around the $80 \mathrm{kGy}$ irradiated sample, then increases with increasing the electron beam dose up to 200 kGy. The melting temperature $T_{m}$ is sensing the crystalline domains of the polymer. It is possible to speculate that at low doses (10-80 kGy), defects generation splits the crystals depressing the melting temperature. For such doses, the decrease of the polymer length contributes also to the shift of $T_{m}$ towards lower temperatures. At higher doses (80-200 kGy), the thickness of crystalline structures (lamellae) is increased.

\subsection{Color changes :}

The transmission spectra of CTA samples in the wavelength range 200-2500 nm have been investigated. The spectra appeared, for all CTA samples, as a band with different intensities. The color intercepts $\left(L^{*}, a^{*}\right.$, and $\left.b^{*}\right)$ before and after exposure are shown in Table 2. The accuracy in measuring $L^{*}$ is \pm 0.05 and \pm 0.01 for $a^{*}$ and $b^{*}$. It can be seen that the color parameters were totally changed after exposure to electron beam irradiation. The red $\left(+a^{*}\right)$ color component of the non-irradiated film was changed to green $\left(-a^{*}\right)$ after exposure to electrons in the dose range (130-200 kGy).

The color intensity $\Delta E^{*}$, differences between the non-irradiated and irradiated samples was calculated, given in Table 2 and plotted in Figure 3 as a function of electron dose.

Table 2. The color intercepts $\left(L^{*}, a^{*}\right.$, and $\left.b^{*}\right)$ and color intensity $\Delta E^{\star}$ of CTA samples as a function of the electron dose.

\begin{tabular}{lcccc}
\hline \multirow{2}{*}{$\begin{array}{l}\text { Dose } \\
\text { (kGy) }\end{array}$} & \multicolumn{3}{c}{ Color intercepts } & $\Delta E^{*}$ \\
\cline { 2 - 4 } & $L^{*}$ & $a^{*}$ & $b^{*}$ & \\
\hline 0 & 34.4 & 0.58 & 3.8 & 0.00 \\
10 & 33.5 & 0.46 & 3.3 & 1.65 \\
20 & 34.2 & 0.37 & 3.87 & 3.32 \\
40 & 33.7 & 0.08 & 5.3 & 2.53 \\
80 & 34.3 & 0.04 & 4.82 & 3.25 \\
100 & 33.1 & 0.02 & 5.36 & 4.47 \\
130 & 33.4 & -0.32 & 7.26 & 4.42 \\
160 & 33.4 & -0.24 & 7.56 & 4.54 \\
200 & 33.0 & -0.26 & 8.10 & 5.00 \\
\hline
\end{tabular}




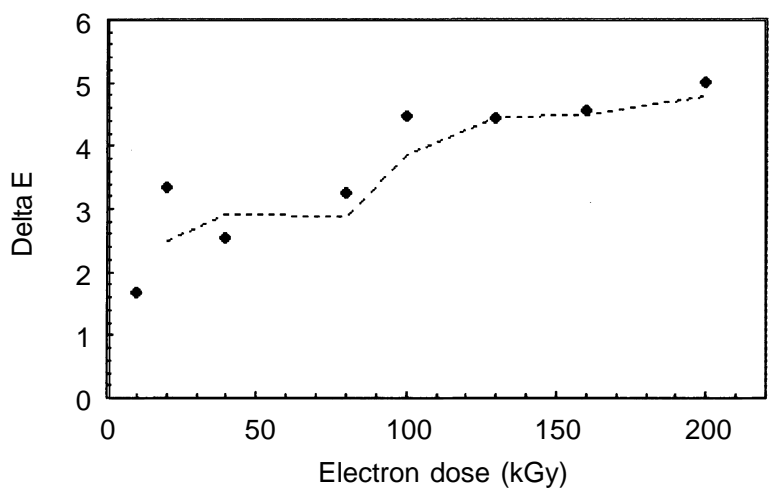

Figure 3. Variation of the color intensity $\Delta E^{*}$ (color differences between the non-irradiated and irradiated CTA samples) with the electron dose.

The color intensity $\Delta E^{*}$ was greatly increased with increasing the electron beam dose, and accompanied with a significant increase in the blue color component $\left(b^{*}\right)$. This indicates that the CTA polymer has more response to color change by electron beam irradiation. These changes in color can be attributed to the trapping of the excited free radicals that are formed by ionization. Also, the trapped free radicals resulting from radiation-induced rupture of polymer molecules have electrons with unpaired spin. Such species may also give optical coloration.

\section{Conclusion}

The irradiation of cellulose triacetate polymer in the dose range 80-200 kGy shifts the temperature at which the decomposition starts to higher values indicating higher thermal stability.

At low doses (10-80 kGy), defects generation splits the crystals depressing the melting temperature, while at higher doses (80-200 kGy), the thickness of crystalline structures (lamellae) is increased, thus the melting temperature increases.

The non-irradiated cellulose triacetate is nearly colorless; however, it showed significant color sensitivity towards electron beam irradiation. The sensitivity in color change towards electron beam irradiation appeared clearly in the change in red color component for the non-irradiated CTA film into green and also in the increase of the blue color component.

\section{References}

[1] S Ryszard, Z Danuta, M Wanda, P Dominik and Gfóarf Radial Phys. Chem. 75259 (2006)

[2] H Khan and I Qureshi Radiat. Meas. 3125 (1999)

[3] S Nouh and T Hegazy Radiat. Meas. 4117 (2006)

[4] S Nouh, L Wahab and H Essia Radiat. Meas. 30753 (1999)

[5] J Chen, M Czayka and R Uribe Radial Phys. Chem. 7431 (2005)

[6] T Ryuichi, M Shoichi and T Naoyuki Intl. J. Appl. Radiat. Isoto 35875 (1984) 
[7] S Nouh Radiat. Meas. 27499 (1997)

[8] M Senna, H Abdel-Hamid and M Hussien Nucl. Instr. Meth. B187 48 (2002)

[9] L Woo and C Sandford Radiat. Phys. Chem. 63845 (2002)

[10] R Mishra, S Tripathy, K Dwivedi, D Khathing, S Ghosh, M Muller and D Fink Radiat. Meas. 37247 (2003)

[11] P Samuel, K Toshiyuki, S Kimio, A Satoshi, M Hideto, T Masaaki and S Toshio J. Membrane Science 244 251 (2004)

[12] Z Ali, M Hossam and H Said Radiat. Phys. Chem. 7553 (2006)

[13] C Aymes, N Betz, B Legendre and N Yagoubi Polym. Degrad. Stabil. 91649 (2006)

[14] H Khonakdara, S Jafarib, U Wagenknechtc and D Jehnichenc Radiat. Phys. Chem. 7578 (2006)

[15] K Nassau Color for Science, Art and Technology (New York : Elsevier) (1998)

[16] M Hossam, Z Ali and E Hussein J. Appl. Polym. Sci. 1014358 (2006)

[17] H Horowitz and G Metzger Analytical Chemistry 351464 (1963) 